\title{
Refined description of momentum transfer in real liquid mixtures
}

\author{
(C) Arnold Sh. Bicbulatov, ${ }^{1}$ and Asia A. Usmanova ${ }^{2+}$ \\ ${ }^{1}$ Department of Chemical Engineering Apparatus. Kazan National Research Technological University. \\ Siberian Ave., 68. Kazan, 420029. Tatarstan Republic. Russia. \\ Phone:+7 (843) 231-40-32.E-mail: iraida@kstu.ru \\ ${ }^{2}$ Department of Automation Sistems and Prosess Control. Kazan National Research Technological \\ University. Karl Marx St., 72. Kazan, 420015. Tatarstan Republic. Russia. \\ Phone: +7 (843) 231-42-72.E-mail: sautp@yandex.ru
}

\begin{abstract}
*Supervising author; ${ }^{+}$Corresponding author
Keywords: viscosity coefficients, nonideal liquid mixtures, kinetic theory hart spheres model, thermodynamics of irreversible processes, activity coefficients.
\end{abstract}

\begin{abstract}
Viscosity coefficients are required to calculate various heat and mass transfer processes. There is not strict theory that allows calculating viscosity coefficients in real systems. The kinetic theory of dense media for the model of solid sphere, which takes into account only the presence of its own volume of particles, allows us to calculate the transfer coefficients in ideal mixtures. A real mixture of different events the forces of attraction play a significant role in their characteristic behavior at different concentrations of the mixture. The problem of taking into account the real interaction between molecules can be solved using methods of thermodynamics irreversible processes. The formalism of such a solution of kinetic equations is based on a model representation of the behavior of molecules in real systems when indirectly obtaining information about a specific interaction of particles through the value of the chemical potential or activity. This work is a continuation of the development of other published works. To account for the interaction between molecules in real systems, a thermodynamic model of an ideal associated Prigogine solution is used. This model assumed that non-ideal systems can represented as ideal under certain conditions and assumes that attractive forces can act between particles. It is possible to form complexes and their interaction. In accordance with the definition of the chemical potential introduced by Lewis, real systems with particle density $n_{i}$ are replaced by an equivalent ideal mixture with activity $a$. In this paper, we refine the generalized kinetic equations of dense media used as an ideal comparison system for real solutions. A new expression for the flow term in the kinetic equation is obtained.
\end{abstract}

\section{References}

[1] A.S. Bikbulatov. Kinetic description of diffusion in non-ideal multicomponent systems. Zhurn. Physical. Chemistry. 1987. Vol.LXI. No.7. P.2166-2170. (russian)

[2] A.S. Bikbulatov. Modified description of energy transfer in non-ideal liquid multicomponent systems. Zhurn. physical. chemistry. 1998. Vol.72. No.82. P.1517-1519. (russian)

[3] A.S. Bikbulatov. Zhurn. Physical. Chemistry. 1995. Vol.19. No.2. P.362. (russian)

[4] I. Prigogine, R. Defay. Chemical thermodynamics. Novosibirsk. 1966. P.508. (russian)

[5] D. Ferziger, G. Kaper. Mathematical theory of the transfer process in gases. Moscow: World. 1976. 554p. (russian)

[6] Kinetic Theory of Multicomponent Dense Fluid Mixtures of Rigid Spheres. M.K. Tham, K.E. Gubbins. Zhurn. Physical. Chemistry. 1971. Vol.55. No.1. P.268-279.

[7] J.H. Kincaid. Ithotermal diffusion in binary hard sphere mixtures the effects of the Onsager visiprosity relation. Phys. Let. 1978. Vol.64. No.5. P.429-432. 